\title{
Relações sociais, cognição na doença de Alzheimer: revisão sistemática
}

\author{
Gabriela Cabett Cipolli \\ Deusivania Vieira da Silva Falcão \\ Universidade de São Paulo, SP, Brasil
}

\section{Resumo}

O objetivo deste estudo foi realizar uma revisão sistemática sobre estudos acerca das relações sociais, cognição na doença de Alzheimer. Foi realizada uma busca nas bases de dados PubMed, LILACs, BIREME e SCOPUS, utilizando os termos "Alzheimer" and "social relationships". O banco final incluído na análise foi composto por 15 artigos, publicados desde 2007. Foi constatada a necessidade de um incremento na realização de pesquisas que visem a compreender como o suporte social se desenvolve e é vivenciado por idosos no contexto brasileiro, sugerindo direções futuras para o desenvolvimento de estudos nessa área no país.

Palavras-chave: Relações sociais; Idosos; Doença de Alzheimer; Cognição.

\section{Social relations, cognition in Alzheimer's disease: systematic review}

\begin{abstract}
The objective of this study was to perform a systematic review onstudies about social relationsas a predictor factor for cognitive decline and Alzheimer's disease. A search was performed on the PubMed, LILACs, BIREME and SCOPUS databases using the terms "Alzheimer" and "social relations". The final bank included in the analysis of 15 articles, published since 2007. The researchers found indicated that having a good quality in the social relations are considered important to maintain the cognition preserved. In addition, it was that the necessity of an increment of researches that aim to understand how the social support develops and is experience by the elderly in the Brasilin context, suggesting futures directions for the development of studies in this area of the country.
\end{abstract}

Keywords: Social relations; Old people; Alzheimer's disease; Cognition.

\section{Las relaciones sociales, la cognición en la enfermedad de Alzheimer: revisión sistemática}

\section{Resumen}

El objetivo de este estudio fue realizar una revisión sistemática de los estudios sobre el relaciones sociales como predictor de deterioro cognitivo y enfermedad de Alzheimer. Se realizó una búsqueda en las bases de datos PubMed, Lilacs, BIREME y Scopus utilizando los términos de "Alzheimer" y "relaciones sociales". La base de datos final incluidos en el análisis consistió en 15 artículos publicados desde 2007. La investigación encontró indicó que tener una buena calidad de las relaciones sociales se consideran importantes para el mantenimiento de la cognición conserva. Además, la necesidad de un aumento en la realización de la investigación dirigida a la comprensión de cómo el apoyo social fue encontrado desarrolla y es experimentado por las personas mayores en el contexto brasileño, lo que sugiere direcciones futuras para el desarrollo de los estudios en esta área en el país.

Palabras clave: Las relaciones sociales; Los ancianos; Enfermedad de Alzheimer; Cognición. 


\section{Introdução}

A população mundial está envelhecendo e dentre as alterações observadas que ocorrem com o envelhecimento, as que certamente exigem investigações incluem as alterações cognitivas(Aprahamian, Stella, \& Forlenza, 2013; Gallagher, Luck, \& Del Vecchio, 2010). Segundo o World Alzheimer Report (2009), atualmente, mais de 46 milhões de pessoas no mundo vivem com algum tipo de demência, e a cada ano surgem cerca de nove milhões de novos casos. Segundo a Organização Mundial de Saúde (World Health Organization, 2012) a doença de Alzheimer (DA) é o tipo mais comum de demência. Observa-se que as perdas cognitivas aumentam invariavelmente, e o indivíduo progressivamente tanto perde a memória quanto a autonomia, até permanecer dependente de outros para a execução de tarefas básicas. A DA é associada com a progressão do prejuízo no comportamento social e no funcionamento, embora o conceito de prejuízo social não esteja claramente definido nos critérios de diagnóstico clínico de demência (Jing, Willis, \& Feng, 2016; Wortmann, 2012).

O envelhecimento de um modo geral é tido como inevitavelmente acompanhado por uma perda progressiva dos laços de apoio dos membros da família e dos amigos, havendo uma tendência de diminuição da rede social, designada como o conjunto de relações sociais que um indivíduo mantém, incluindo as características dessas relações. Os contatos sociais e os relacionamentos são as maneiras pelas quais as pessoas se influenciam mutuamente e influenciam seu ambiente (Bowling, 1991). A definição e a operacionalização no que se refere às relações sociais diferem entre os estudos, dificultando a integração dos resultados das pesquisas publicadas. As relações sociais podem, por exemplo, conter características estruturais, como o número de laços sociais, mas, também, aspectos qualitativos, por exemplo, os níveis de suporte social e a qualidade dessas relações (Kuiper et al., 2015).

As relações sociais que um sujeito apresenta ou desenvolve ao longo de seu ciclo vital guardam entre si relações hierárquicas, conferindo-lhes características de rede. O grau de significado da rede de relações sociais relaciona-se à avaliação que a pessoa faz sobre a natureza das suas relações (ex: formais ou informais, com familiares ou amigos, íntimas ou distantes) e ao grau em que elas suprem as suas necessidades. Nesse sentido, avalia-se o tipo de interação que proporcionam (ex: afetivas, informativas ou instrumentais) e o grau de desejabilidade dos relacionamentos e das interações (ex: de livre escolha, compulsórias, funcionais e disfuncionais) (Capitanini, 2000).
As relações sociais e as atividades significativas são fatores importantes para o envelhecimento bemsucedido, bem-estar subjetivo e saúde percebida ao longo da vida (Rowe \& Kahn, 1998). O envolvimento frequente nas relações sociais está associado a melhor saúde mental e física, bem como a redução de risco da mortalidade (Tay, Tan, Diener, \& Gonzalez, 2013). O tipo de interação social e o laço que se tem com outras pessoas se estendem como fatores de risco ou proteção ao bem-estar do idoso, favorecendo ou não as habilidades para o enfrentamento de doenças. Nessa direção, um suporte social adequado pode beneficiar a saúde através da redução do estresse, fornecendo recursos psicológicos e materiais necessários para lidar com os estressores (Kuiper et al., 2015). O suporte social tem sido definido como relações com outras pessoas que são amadas e cuidadas, estimadas e valorizadas, e que fazem parte de uma rede de comunicação e obrigações mútuas. Ela pode vir de um cônjuge ou companheiro, parentes, amigos, colegas de trabalho e laços comunitários (Kim, Sherman, \& Taylor, 2008; Gomes, 2016).Além disso, evidências mostraram que quando os indivíduos apresentam recursos sociais maiores e de boa qualidade, como uma rede de amizade maior, está relacionada à melhora do funcionamento da memória em adultos mais velhos (Sörman et al., 2015). Os efeitos de estimulação intelectual e social inerente a uma relação conjugal, por exemplo, diminuem as chances de se ter uma deterioração cognitiva na velhice (Falcão, 2016).

A perda de relações sociais em idosos com demências tende a ser mais rápida devido aos danos cognitivos e aos estigmas encontrados em relação às demências, principalmente, a doença de Alzheimer. No entanto, alguns estudos mostraram que se tivermos e/ou mantermos a rede de relações sociais na velhice, o suporte poderia ser protetor conta o comprometimento cognitivo leve (CCL) e DA leve (Amieva et al., 2010). Pesquisas na área da gerontologia vêm demonstrando efeitos mistos do apoio social sobre o declínio cognitivo e a demência: o apoio social tem se mostrado protetor em alguns estudos, mas não em outros. Ademais, pouco se sabe sobre os mecanismos subjacentes entre o apoio social e o funcionamento cognitivo. $\mathrm{O}$ Longitudinal Aging Study Amsterdam (LASA) incluiu 2255 participantes holandeses com idade entre 55-85 anos.Constatou-se que o frequente suporte emocional das relações sociaisfoi relacionado aos sentimentos reduzidos de solidão e melhor funcionamento cognitivo. Acréscimo no apoio emocional também aumentaram diretamente o desempenho cognitivo. $\mathrm{O}$ efeito protetor do apoio emocional foi mais forte entre os adultos com 65 anos e mais velhos. Por outro 
lado, o aumento no apoio instrumental não amorteceu o declínio cognitivo, em vez disso, houve indicações de declínio mais rápido, concluindo-se que as relações de apoio emocional são um protetor mais poderoso do declínio cognitivo do que as relações de apoio instrumental (Ellwardt, Aartsen, Deeg, \& Steverink, 2013).

Conforme a Teoria da Seletividade Socioemocional (Carstensen, 1991) o envelhecimento implica que os mais velhos fiquem mais seletivos e apenas incluam na sua rede as pessoas emocionalmente importantes, tendendo a perceber mais positivamente sua vida quando estão envoltas de pessoas significativas no seu dia-a-dia (Charles \& Carstensen, 2010). Quando os indivíduos são mais jovens estão mais propensos a priorizar os objetivos de aquisição de novas informações, procurando experiências novas ou expandir as redes sociais. No entanto, à medida que envelhecem, percebem o tempo futuro como sendo mais limitado. Assim sendo, o foco motivacional desloca-se para a busca do bem-estar emocional e do sentido da vida. As limitações de tempo percebidas, e não a idade em si leva as pessoas a buscarem parceiros emocionalmente próximos em relação aos parceiros sociais periféricos (Fung \& Carstensen, 2006).

Partindo-se dessas informações, o presente estudo teve como objetivo realizar uma revisão sistemática da literatura sobre as relações sociais e a cognição na doença de Alzheimer. O intuito foi compreender como os pesquisadores têm investigado esse assunto, quais tipos de método têm sido adotados e os principais resultados encontrados. Este estudo poderá contribuir para uma melhor compreensão acerca dessa temática, refletindo como esse fator pode influenciar o bem-estar dos idosos e seus familiares. Além disso, favorecerá a elaboração de propostas de intervenções que promovam habilidades sociais e o fortalecimento das relações.

\section{Método}

Realizou-se, inicialmente, uma busca por artigos indexados nas bases de dados eletrônicas PubMed, LILACs, BIREME, PsycInfo e SCOPUS, considerando o período de janeiro de 2006 a agosto de 2016. A busca foi feita entre os meses de outubro, novembro e dezembro do ano de 2016. Foram realizadas as combinações dos seguintes descritores e suas respectivas traduções em português e em espanhol, em cada uma das bases selecionadas: "Alzheimer" e "social relationships". Como operadores foi utilizado o "AND”. Em um primeiro momento, foram excluídos os artigos duplicados entre as bases consultadas. Em seguida, baseando-se na análise dos resumos, foram selecionados apenas os estudos que atenderam aos seguintes critérios de inclusão: (1) ter a presença dos descritores; (2) incluir idosos de 60 anos e mais ou média de idade dentro desse intervalo; (3) a amostra ser composta por ambos os sexos ou apenas um sexo; (4) artigos disponíveis na íntegra pelo Sistema Integrado de Bibliotecas da Universidade de São Paulo (SIBi USP); (5) ser artigo original de pesquisa desenvolvida com seres humanos, publicado em periódico indexado nas bases utilizadas, em inglês/ português/espanhol, durante janeiro de 2006 a agosto de 2016.Artigos de revisão, monografias, dissertações, teses, resumos, capítulos ou livros e ponto de vista/ opinião de especialistas não foram incluídos. Também foram excluídos os estudos com adultos, adolescentes e crianças, além de artigos relacionados com tipos de medicamentos entre outros fatores biológicos.

A busca inicial nas bases de dados gerou um total de 779 artigos (Pubmed: 388; LILACS: 29; Bireme: 110; Scopus: 252). Na primeira triagem, foram excluídos 351 com base na análise do título. Dos 428 artigos restantes, 408 não atenderam aos critérios de inclusão ou não se teve acesso ao artigo na íntegra. Restaram, portanto, 20, porém, 10 foram excluídos por estarem duplicados entre as bases. No que diz respeito às estratégias complementares, a busca nas listas de referência teve como resultado a inclusão de cinco trabalhos. $\mathrm{O}$ pesquisador consultado, por sua vez, considerou o resultado da revisão correto e não fez nenhum acréscimo. Assim, o banco final incluído na análise deste estudo foi constituído por 15 artigos (ver Figura 1), destacados com asterisco na lista de referências.

Foi feita uma análise minuciosa, na íntegra, dos artigos que atenderam aos critérios de inclusão por dois avaliadores de forma independente. Nos casos em que houve discordância inicial, foi realizada uma nova análise conjunta até ser encontrado consenso. Em seguida, os artigos foram agrupados em quatro diferentes categorias, a saber: (1) País de origem; (2) Tipo de estudo (metodologia de pesquisa quantitativa, pesquisa qualitativa, método misto); (3) Contexto (comunidade, instituições de cuidados) e; (4) Principais achados no que se refere às relações e cognição na doença de Alzheimer.

\section{Resultados}

Pesquisas (Fankhauser et al., 2015;Wilson et al., 2015) indicaram que o suporte social diminuído parece estar envolvido com o declínio cognitivo e demência incidente e do tipo Alzheimer em idosos que apresentavam interação social negativa ou com frequência baixa na proximidade com seus 


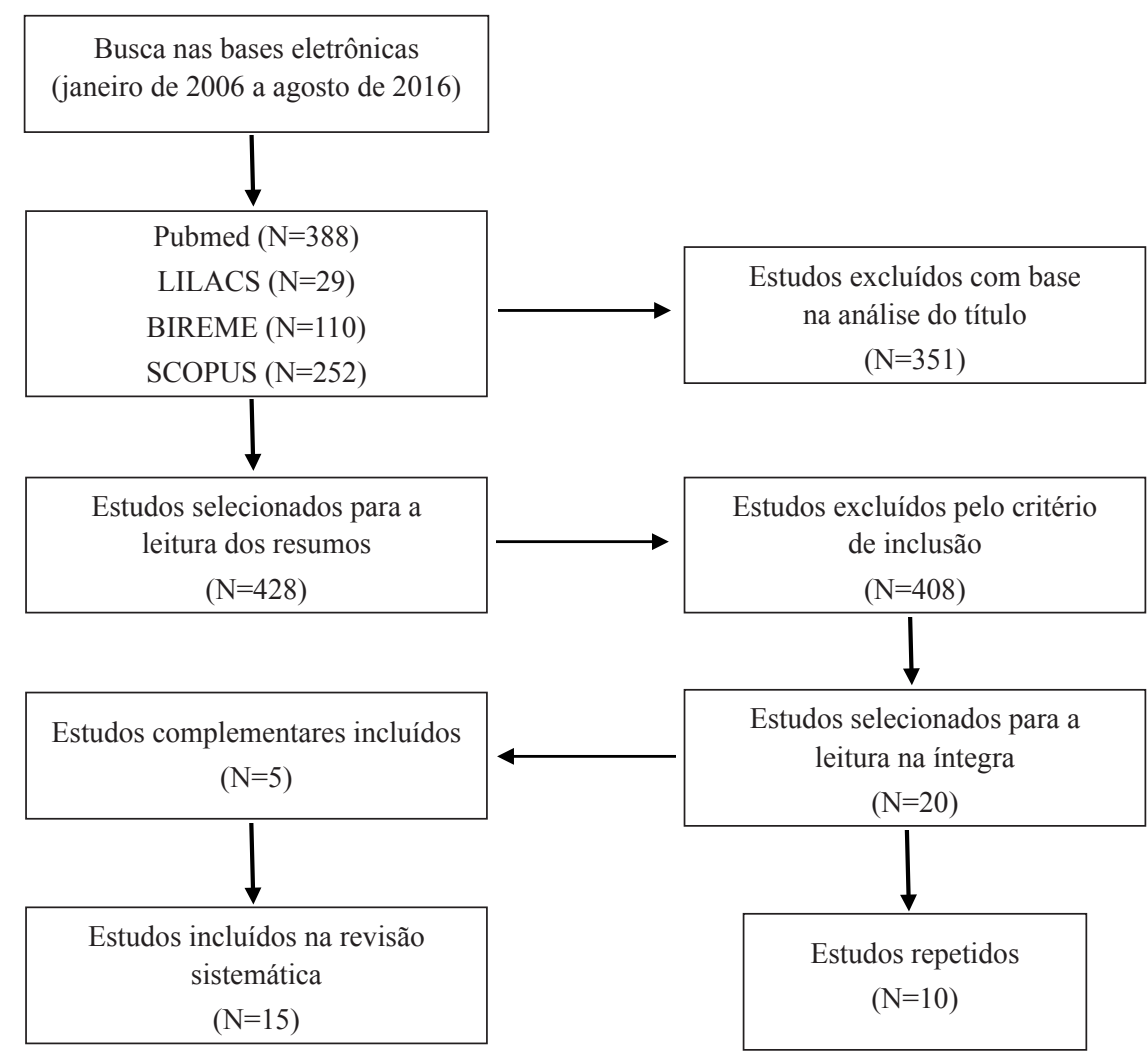

Figura 1. Etapas do processo de revisão sistemática

contatos (Tabela 1). Um estudo prospectivo e de coorte realizado na Suécia mostrou as associações entre aspectos das relações sociais em idosos e demência incidente. Observou-se que quanto maior o índice de relacionamento menor o risco das causas de demências e de DA, tanto antes como após o controle das covariáveis, incluindo estresse e sintomas depressivos. Algumas variáveis de relação social (que visitam/visitas de amigos e conhecidos uma vez por semana ou mais) foram relacionadas a um menor risco de todas as causas de demência (Sörman, Rönnlund, Sundström, Adolfsson, \& Nilsson, 2015). No entanto, as relações sociais e a rede social são variáveis complexas, quando se considera a influência delas na proteção potencial contra a doença de Alzheimer e outros tipos de demência. Não ficaram claro quais características da rede social são responsáveis para essa influência positiva (Amieva et al., 2010).

Fankhauser et al. (2015) avaliou o impacto dos processos motivacionais baseados em ocupação e variáveis de redes sociais sobre a incidência de demência mais de oito anos após o estudo. Constatou-se que os indivíduos os quais foram diagnosticados com doença de Alzheimer na avaliação de acompanhamento, não tiveram frequência significativamente menor de contatos sociais ou menor proximidade de contatos sociais do que aqueles que não foram diagnosticados com demência. No entanto, confirmou-se que a frequência dos contatos sociais é um preditor significativo, ou seja, a maior frequência de contato social está associada com o menor risco de demência. Já a proximidade de contatos sociais não teve impacto significativo sobre o risco de demência. Numa pesquisa em que foi investigada a participação social em idosos vivendo em casa com doença de Alzheimer leve foi possível identificar os preditores de baixa participação social. A principal descoberta foi que o prejuízo social está presente no estágio leve da doença de Alzheimer. Assim sendo, a baixa participação social está presente na DA leve e os preditores independentes significativos de baixa participação social foram prejudicados nas atividades de vida diária (AVD) e sintomas neuropsiquiátricos. Os resultados sublinharam a importância de avaliar a participação social na DA leve, uma vez que são as poucas habilidades AVDs perdidas (Sørensen, Waldorff, \& Waldemar, 2008).

Além disso, os sintomas depressivos e engajamento social têm sido associados com risco de comprometimento cognitivo leve (CCL) e relacionados com pontuação negativa de interação social (Wilson et al., 2015). Nesse mesmo estudo detectou-se que as interações sociais negativas crônicas podem ser um fator de 
risco para o CCL e declínio cognitivo em idade avançada. Desta maneira, os resultados não só sugeriram que as interações sociais negativas estavam associadas com o declínio cognitivo da idade avançada, mas também enfatizaram a importância clínica desta associação, mostrando que eles prevêem o desenvolvimento do CCL, amplamente reconhecido como o precursor de demência. Complementando esse achado, Sörman et al. (2015) investigaram a associação entre cada variável referente às relações sociais e demência. Os pesquisadores demonstraram com base em todo o período de acompanhamento do estudo que o valor mais alto no índice de relacionamento foi associado com risco reduzido de todas as causas de outras demências e DA, antes e após o controle para as covariáveis, incluindo o estresse e sintomas depressivos.

No que se refere às relações intergeracionais, pesquisadores (Celdrán, Triadó, \& Villar, 2009) investigaram a interação social entre netos adolescentes (média de 17.69 anos) e avós, tanto preservados cognitivamente quanto aqueles com demência. Os aspectos relacionados com fatores emocionais da relação avô-neto, como a proximidade ou satisfação com o relacionamento, foram significativas maiores nos avôs cognitivamente preservados. Esta diferença pode estar nos sintomas clássicos de demência, tais como perda de memória, problemas com a linguagem ou o reconhecimento, o que impediria os papéis mais simbólicos (portador da história da família, ser o confidente dos problemas dos adolescentes, entre outros) que os avôs podem fazer quando seus netos são adolescentes ou adultos jovens e isso envolveria menor percepção e menor proximidade com os avôs.

Em contrapartida as redes sociais dependem tanto de características estruturais das pessoas com quem se compartilham as relações sociais e a percepção que se tem da qualidade do apoio que são fornecidos pelos membros de sua rede. Uma questão interessante é se as características estruturais da rede (por exemplo, o tamanho, a frequência de contatos), ou as características de apoio (por exemplo, apoio emocional percebido, satisfação com as interações) ou ambos têm um impacto significativo sobre o desenvolvimento do processo de demência (Crooks, Lubben, Petitti, Little, \& Chiu, 2008). Essa questão foi particularmente refletida pelo estudo de Amieva et al.(2010), em que as únicas variáveis associadas à demência subsequente ou a doença de Alzheimer eram as que refletiam a qualidade das relações sociais. Os idosos participantes que se sentiam muito satisfeitos com a sua rede social tiveram $23 \%$ menos risco de desenvolver demência de 5 a 15 anos após o estudo, enquanto os participantes que achavam que eles tinham recebido de outros mais do que deram a eles tiveram $55 \%$ menos risco de demência subsequente e risco reduzido de $53 \%$ para a doença de Alzheimer.

TABELA 1

Resultado da Pesquisa, em ordem decrescente de publicação

\begin{tabular}{|c|c|c|c|c|c|c|c|}
\hline \multirow{2}{*}{ Autor/Ano } & \multicolumn{2}{|c|}{ Amostra } & \multirow{2}{*}{$\begin{array}{l}\text { Instrumentos para } \\
\text { avaliação das } \\
\text { relações sociais }\end{array}$} & \multirow{2}{*}{ Local } & \multirow{2}{*}{ Tipo de estudo } & \multirow{2}{*}{ Contexto } & \multirow{2}{*}{ Principais achados } \\
\hline & $N$ & Idade & & & & & \\
\hline Jones, D (2015) & 1 & 72 & $\begin{array}{l}\text { Conversation } \\
\text { analysis }\end{array}$ & Reino Unido & $\begin{array}{l}\text { Estudo de } \\
\text { Caso }\end{array}$ & Instituição & $\begin{array}{l}\text { As dificuldades de pacientes } \\
\text { com doença de Alzheimer } \\
\text { em se comunicar afetam as } \\
\text { relações sociais }\end{array}$ \\
\hline Smit et al (2015) & 1144 & 84,2 & $\begin{array}{l}\text { RAI-MDS; } \\
\text { QUALIDEM }\end{array}$ & Holanda & Transversal & Instituição & $\begin{array}{l}\text { Relação negativa entre } \\
\text { o envolvimento social, } \\
\text { das atividades sociais } \\
\text { desenvolvidas e a } \\
\text { autoimagem positiva }\end{array}$ \\
\hline Clarke et al (2015) & 6158 & 74 & $\begin{array}{l}\text { Chicago Health and } \\
\text { Aging Project }\end{array}$ & Chicago & Longitudinal & Comunidade & $\begin{array}{l}\text { O envolvimento com } \\
\text { a vizinhança e com os } \\
\text { recursos da comunidade } \\
\text { diminui o declínio cognitivo } \\
\text { em idosos }\end{array}$ \\
\hline Wilson et al (2015) & 529 & 81,4 & $\begin{array}{l}\text { Social network size } \\
\text { was the number of } \\
\text { these individuals } \\
\text { seen at least once a } \\
\text { month, as reported } \\
\text { elsewhere }\end{array}$ & Chicago & Longitudinal & Comunidade & $\begin{array}{l}\text { Interações sociais negativas } \\
\text { podem ser um fator de risco } \\
\text { para transtorno cognitivo } \\
\text { leve e declínio cognitivo em } \\
\text { idade avançada }\end{array}$ \\
\hline
\end{tabular}


TABELA 1 (conclusão)

\begin{tabular}{|c|c|c|c|c|c|c|c|}
\hline \multirow{2}{*}{$\begin{array}{l}\text { Autor/Ano } \\
\text { Wang, He \& Dong } \\
\text { (2015) }\end{array}$} & \multicolumn{2}{|c|}{$\begin{array}{l}\text { Amostra } \\
N \text { Idade }\end{array}$} & \multirow{2}{*}{$\begin{array}{l}\text { Instrumentos para } \\
\text { avaliação das } \\
\text { relações sociais } \\
\text { Project of Longevity } \\
\text { and Ageing in } \\
\text { Dujiangyan, China }\end{array}$} & \multirow{2}{*}{$\begin{array}{l}\text { Local } \\
\text { Dujiangyan } \\
\text { (China) }\end{array}$} & \multirow{2}{*}{$\begin{array}{l}\text { Tipo de Estudo } \\
\text { Transversal }\end{array}$} & \multirow{2}{*}{$\begin{array}{l}\text { Contexto } \\
\text { Comunidade }\end{array}$} & \multirow{2}{*}{$\begin{array}{l}\text { Principais Achados } \\
\text { Ser solteiro, não ter amigos } \\
\text { e baixo nível de contato } \\
\text { social estão associados com } \\
\text { aumento do risco de declínio } \\
\text { cognitivo }\end{array}$} \\
\hline & 764 & 93,4 & & & & & \\
\hline $\begin{array}{l}\text { Fankhauser et al. } \\
\text { (2015) }\end{array}$ & 772 & 81,21 & O*NET; PANT & Alemanha & Longitudinal & Comunidade & $\begin{array}{l}\text { A frequência e a } \\
\text { proximidade dos contatos } \\
\text { sociais têm um impacto } \\
\text { diferencial no risco de } \\
\text { demência }\end{array}$ \\
\hline $\begin{array}{l}\text { Sörman et al. } \\
(2015)\end{array}$ & 1769 & 74,69 & $\begin{array}{l}\text { They were } \\
\text { obtained through } \\
\text { questionnaires and } \\
\text { interviews conducted } \\
\text { by nurses }\end{array}$ & Suécia & Longitudinal & Comunidade & $\begin{array}{l}\text { Aspectos das relações } \\
\text { sociais estão associados à } \\
\text { demência incidente ou DA }\end{array}$ \\
\hline $\begin{array}{l}\text { Farrell et al. } \\
(2014)\end{array}$ & 236 & 76 & $\begin{array}{l}\text { Pleasant Events } \\
\text { Schedule-AD } \\
\text { (PES-AD) }\end{array}$ & EUA e França & Longitudinal & Comunidade & $\begin{array}{l}\text { Indivíduos com DA que tem } \\
\text { falhas de apuramento de } \\
\text { palavra são menos propensos } \\
\text { a participar e desfrutar de } \\
\text { atividades sociais e de lazer }\end{array}$ \\
\hline $\begin{array}{l}\text { Ellwardt et al. } \\
(2013)\end{array}$ & 2255 & 63,45 & $\begin{array}{l}\text { Pre-set questionnaire } \\
\text { form }\end{array}$ & Amsterdam & Longitudinal & Comunidade & $\begin{array}{l}\text { O efeito protetivo do suporte } \\
\text { emocional torna-se forte em } \\
\text { pessoas com } 65 \text { anos e mais } \\
\text { velhos }\end{array}$ \\
\hline $\begin{array}{l}\text { Amieva et al. } \\
(2010)\end{array}$ & 2089 & 73,7 & $\begin{array}{l}\text { Was obtained by } \\
\text { personal interview } \\
\text { by trained nurses at } \\
\text { baseline }\end{array}$ & França & Longitudinal & Comunidade & $\begin{array}{l}\text { As únicas variáveis } \\
\text { associadas à demência ou } \\
\text { à doença de Alzheimer } \\
\text { foram aquelas que refletem } \\
\text { a qualidade das relações } \\
\text { sociais }\end{array}$ \\
\hline $\begin{array}{l}\text { Andrew \& Rockwood } \\
(2010)\end{array}$ & 2468 & 79,1 & $\begin{array}{l}\text { Selfreport variables } \\
\text { relating to potential } \\
\text { social deficits }\end{array}$ & Canadá & Longitudinal & Comunidade & $\begin{array}{l}\text { O aumento da } \\
\text { vulnerabilidade social foi } \\
\text { associado ao maior declínio } \\
\text { cognitivo }\end{array}$ \\
\hline $\begin{array}{l}\text { Conroy et al. } \\
(2010)\end{array}$ & 802 & 74,2 & $\begin{array}{l}\text { Aging Research } \\
\text { Programme (HARP) }\end{array}$ & $\begin{array}{l}\text { República da } \\
\text { Irlanda }\end{array}$ & Transversal & Comunidade & $\begin{array}{l}\text { A solidão e a propensão } \\
\text { ao tédio estão associadas à } \\
\text { redução da função cognitiva } \\
\text { em idade avançada }\end{array}$ \\
\hline $\begin{array}{l}\text { Montserrat Celdrán et al. } \\
\text { (2008) }\end{array}$ & 64 & 77,35 & $\begin{array}{l}\text { Cuestionario que } \\
\text { hacia referencia a } \\
\text { cinco aspectos de } \\
\text { la relación entre los } \\
\text { abuelos y sus nietos; } \\
\text { GDS }\end{array}$ & Espanha & Qualitativo & Comunidade & $\begin{array}{l}\text { A demência envolve } \\
\text { transformação na relação } \\
\text { entre avós e netos que } \\
\text { vão além das mudanças } \\
\text { que podem observar } \\
\text { normativamente }\end{array}$ \\
\hline $\begin{array}{l}\text { Sørensen et al. } \\
(2008)\end{array}$ & 330 & 76,2 & $\begin{array}{l}\text { ADCS-ADL scale; } \\
\text { NPI-Q }\end{array}$ & Dinamarca & Longitudinal & Comunidade & $\begin{array}{l}\text { Importância de avaliar } \\
\text { a participação social na } \\
\text { DA leve, quando poucas } \\
\text { habilidades em AVDs } \\
\text { (Atividades de Vida Diária) } \\
\text { são perdidas }\end{array}$ \\
\hline $\begin{array}{l}\text { Wilson et al. } \\
(2007)\end{array}$ & 823 & 80,7 & $\begin{array}{l}\text { Negative life events } \\
\text { were assessed with } \\
\text { an } 18 \text {-item scale } \\
\text { modified }\end{array}$ & Chicago & Longitudinal & Instituição & $\begin{array}{l}\text { A solidão está associada } \\
\text { com o aumento do risco de } \\
\text { desenvolver DA, mas não é } \\
\text { uma das principais causas }\end{array}$ \\
\hline
\end{tabular}


Clarke et al. (2015) utilizaram dados do Chicago Health and Aging Project, um estudo longitudinal e prospectivo o qual examinaram o papel dos fatores individuais e de vizinhança na trajetória da função cognitiva em idosos. Os participantes tinham 65 anos ou mais e eram residentes na comunidade. Observou-se que os recursos do bairro (ex:. centro de lazer) não foram relacionados aos níveis iniciais da função cognitiva no início da pesquisa. No entanto, a rede de fatores individuais, residência em bairros com recursos da comunidade, a proximidade ao transporte público e espaços públicos em boas condições foram associados com taxas mais lentas de declínio cognitivo, possivelmente por aumentar as oportunidades para atividades físicas e sociais ou para $\mathrm{o}$ acesso a lugares que facilitam o engajamento em atividades.

Conroy et al (2010) refletiram sobre a solidão, a propensão ao tédio, as relações sociais e a depressão que podem estar associadas a função cognitiva em idosos. Foram identificados três grupos de indicadores, a saber: a) baixo apoio social (viúva, viver sozinho e baixo apoio social); b) a reserva cognitiva pessoal (baixa atividade social, nenhum exercício de lazer, não ser casado, solidão e propensão ao tédio) e; c) fatores questões sociodemográficos (ensino primário e se vive em ambiente rural). Na análise realizada, os dois grupos de reserva cognitiva, mas não os apoios sociais foram independentemente associados com a função cognitiva. Já a solidão e a propensão ao tédio foram associadas à redução da função cognitiva em idade avançada, e o grupo com outros fatores associados com reserva cognitiva. Ambos podem ter uma falha comum no mecanismo subjacente para selecionar e manter a atenção sobre as características particulares do ambiente social (solidão) ou para o ambiente nãosocial (propensão ao tédio). Postula-se que a solidão pode afetar adversamente a reserva cognitiva porque os sistemas neurais subjacentes ao comportamento social são menos elaborados e podem compensar menos para outros sistemas neurais comprometidos pela patologia relacionada à idade (Conroy et al., 2010). Em contrapartida Wilson et al (2007) propôs que o isolamento social na velhice pode ser associado com o risco de desenvolvimento de demência. No início do estudo os pesquisadores analisaram 823 idosos institucionalizados em Chicago, e observaram que a solidão foi associada com menor nível de cognição na linha de base e com o rápido declínio cognitivo durante o acompanhamento.

Wang, He \& Dong (2015) analisaram idosos nonagenários e centenários do estudo Project of Longevity and Ageing in Dujiangyan, China e exploraram as associações entre as redes sociais, contatos sociais e o comprometimento cognitivo em pessoas muito idosas. O presente estudo mostrou que o estado civil, número de amigos e níveis de contatos sociais estavam relacionados com o desempenho da função cognitiva entre os nonagenários e centenários chineses. Além disso, observou-seque quanto maior a pontuação no Mini Exame do Estado Mental (MEEM), os chineses apresentavam maior quantidade de amigos e contatos sociais. No entanto, os indivíduos solteiros, com nenhum amigo próximo e baixo nível de contato social foram associados ao aumento do risco de déficit cognitivo nos idosos estudados.

A vulnerabilidade social, definida como um índice que inclui vários fatores sociais está associado com o declínio cognitivo (Andrew \& Rockwood, 2010). Acompanhou-se durante cinco anos idosos canadenses da comunidade para verificar a vulnerabilidade social. Observou-se que o aumento do índice, foi associado com maiores probabilidades de declínio cognitivo. Assim, o estudo concluiu que a vulnerabilidade social está associada ao declínio cognitivo em idosos que vivem em comunidade.

Em estudo longitudinal Farrell et al. (2014) avaliaram a influência da palavra subjetiva e a dificuldade do grau de engajamento em atividades de lazer sociais entre os indivíduos com a doença de Alzheimer. O maior achado dessa pesquisa foi que a dificuldade subjetiva de apuramento de palavra é um importante preditor de envolvimento de atividade social na DA. A falta de avaliação de queixas de linguagem subjetivas pode resultar em sintomas de abstinência sociais, ameaçando a qualidade de vida e o aumento da sobrecarga do cuidador. Concluiu-se que a falta de interação social, pode vir a exacerbar sintomas de linguagem ao longo do tempo. O estudo de Jones (2013) observou que as ligações telefônicas feitas entre a família e idosos com doença de Alzheimer moderada são dificultosas devido ao comprometimento da linguagem nesse estágio da doença, e que a memória semântica ainda pode se manter preservada. Entretanto, a memória episódica estava degenerada dificultando a interação social da família com o idoso e também algumas análises do estudo.

Smit, de Lange, Willemse, Twisk e Pot, (2016) relacionaram o envolvimento em atividades sociais e a qualidade de vida em idosos com demência moderada a grave em Instituição de Longa Permanência. De acordo com as análises, em média, os moradores estavam envolvidos nas atividades para 154 minutos $(\approx 2,5$ horas) no total. Observou-se que o grupo de idosos com demência leve a moderada tivera maior relação 
com o envolvimento e cuidados do que os outros grupos. Também, notou-se que o baixo envolvimento em atividades com a qualidade de vida das subescalas (afeto positivo e relações sociais) era maior do que em outros grupos. No grupo de demência, a relação entre o maior envolvimento e afeto positivo e as relações sociais foi menor do que em outros grupos de status cognitivos. O estudo mostrou que, em geral, o envolvimento em atividades pode ser benéfico para pessoas com demência em relação ao cuidado (aceitar ajudar, não há conflitos com a equipe de cuidados), afeto positivo (é feliz, o humor pode ser influenciado positivamente), inquietação (tensa linguagem corporal), relações sociais (tem contato amigável com outros residentes) e ter algo para fazer (tem coisas a fazer sem a ajuda de outros).

\section{Discussão}

A relação entre relações sociais e cognição na doença de Alzheimer foi demonstrada por todos os estudos incluídos nessa revisão, indicando que indivíduos idosos com relações sociais prejudicadas podem aumentar a probabilidade de declínio cognitivo, e que a frequência e a proximidade dos contatos sociais diminuem esse risco. Entre os estudos que investigaram se há diferença entre o tipo de relação social com outros indivíduos na comunidade ou na instituição aumenta o risco de desenvolver doença de Alzheimer, foi constatado que somente a frequência e a proximidade dos contatos sociais têm um impacto diferencial no risco de doença de Alzheimer e alterações cognitivas. A esse respeito, pode-se pensar que, na atualidade, embora a maioria dos idosos vivam em comunidades exercendo papéis importantes, como a manutenção da rede familiar e de relações sociais, nota-se o acréscimo gradual de doença de Alzheimer e problemas cognitivos. Desse modo, ocupar-se da saúde mental dos idosos, alertando familiares e vizinhos sobre os sinais e sintomas de possíveis alterações cognitivas, que indique a presença da doença de Alzheimer, poderá impedir problemas futuros na relação social do idoso, dentre eles, a diminuição da qualidade das relações e de vida.

Neste estudo foram examinados os resultados de artigos científicos discutindo as relações sociais e cognição na doença de Alzheimer. Existem indícios de que recursos sociais, como uma rede de amizade maior, estão relacionados com um melhor funcionamento da memória em adultos mais velhos (Giles, Anstey, Walker, \& Luszcz, 2012). Além disso, há algumas semelhanças entre os estudos, denotando a correlação entre o baixo nível de suporte social e o aumento da ocorrência de demências. Assim, é importante para os profissionais da área de saúde avaliar o tipo de suporte social em idosos para tentar identificar se existe um possível declínio tanto cognitivo quanto nas AVDs.

Embora pouco se saiba sobre a natureza das relações sociais nas fases da DA, estas podem ser influenciadas pela capacidade intelectual prejudicada, déficits de comunicação e comprometimento funcional. Conforme que a doença progride os idosos com DA são expostos pela distância social (Fratiglioni, Wang, Ericsson, Maytan, \& Winblad, 2000). Consequentemente as relações sociais reduzidas podem expor os pacientes com DA ao declínio cognitivo acelerado e os riscos de saúde adicionais.

\section{Conclusão}

A partir desse estudo, verificou-se que as pesquisas revisadas encontraram associações importantes entre relações sociais e cognição na doença de Alzheimer, ressaltando que as frequências de contatos sociais também podem ser implicadas no aumento da ocorrência de doença de Alzheimer e declínio cognitivo em idosos na comunidade do que em idosos institucionalizados.

A complexidade da rede social de um indivíduo é difícil de compreender. As redes sociais dependem tanto das características estruturais das pessoas com quem se compartilham as relações sociais e a percepção que se tem da própria qualidade do relacionamento e do apoio que os membros da rede podem fornecer. Existem várias razões para pensar que a manutenção de interações sociais é uma das melhores formas de estimulação cognitiva. Este estudo limitou-se a investigar aspectos cognitivos na doença de Alzheimer. Portanto, faz-se mister desenvolver outras pesquisas, como fornecer dados sobre a importância do nível de suporte social e a prevalência de demências, para a melhoria da qualidade de vida dos pacientes e seus cuidadores. 


\section{Referências}

*Amieva, H., Stoykova, R., Matharan, F., Helmer, C., Antonucci, T. C., \& Dartigues, J. F. (2010). What aspects of social network are protective for dementia? Not the quantity but the quality of social interactions is protective up to 15 years later. Psychosomatic Medicine, 72(9), 905-911. https://doi.org/10.1097/PSY.0b013e3181f5e121

*Andrew, M. K. \& Rockwood, K. (2010). Social vulnerability predicts cognitive decline in a prospective cohort of older Canadians. Alzheimer's and Dementia. https://doi.org/10.1016/j.jalz.2009.11.001

Aprahamian, I., Stella, F., \& Forlenza, O. V. (2013). New treatment strategies for Alzheimer's disease: is there a hope? Indian Journal of Medical Research, 138 (October), 449-460.

*Celdrán, M., Triadó, C., \& Villar, F. (2009). Nietos adolescentes con abuelos con demencia: ¿La enfermedad cambia la naturaleza de la relación? Anales de Psicologia.

Charles, S. T. \& Carstensen, L. L. (2010). Social and emotional aging. Annual Review of Psychology, 61, 383-409. https:// doi.org/10.1146/annurev.psych.093008.100448

*Clarke, P. J., Weuve, J., Barnes, L., Evans, D. A., Mendes, C. F., \& Leon, D. (2015). Annals of Epidemiology Cognitive decline and the neighborhood environment. Annals of Epidemiology, 25(11), 849-854. https://doi.org/10.1016/j. annepidem.2015.07.001

*Conroy, R. M., Golden, J., Jeffares, I., Neill, D. O., Conroy, R. M., Golden, J., ..., \& Neill, D. O. (2010). Engagement and depression and their association with cognitive function in older people: a population study, Journal Psychology, Health \& Medicine (December 2016). https://doi.org/10.1080/13548506.2010.487103

Crooks, V. C., Lubben, J., Petitti, D. B., Little, D., \& Chiu, V. (2008). Social network, cognitive function, and dementia incidence among elderly women. American Journal of Public Health, 98(7), 1221-1227. https://oi.org/10.2105/ AJPH.2007.115923

*Ellwardt, L., Aartsen, M., Deeg, D., \& Steverink, N. (2013). Social Science \& Medicine Does loneliness mediate the relation between social support and cognitive functioning in later life? Social Science \& Medicine, 98, 116-124. https:// doi.org/10.1016/j.socscimed.2013.09.002

Fankhauser, S., Forstmeier, S., Maercker, A., Luppa, M., Luck, T., \& Riedel-Heller, S. G. (2015). Risk of dementia in older adults with low versus high occupation-based motivational processes: differential impact of frequency and proximity of social network. J Geriatr Psychiatry Neurol, 28(2), 126-135. https://doi.org/10.1177/0891988714554706

*Farrell, M. T., Zahodne, L. B., Stern, Y., Dorrejo, J., Yeung, P., \& Cosentino, S. (2014). Subjective word-finding difficulty reduces engagement in social leisure activities in Alzheimer's disease. Journal of the American Geriatrics Society. https://doi.org/10.1111/jgs.12850

Figueiredo, M. . G. (2016). Participação social e redes sociais pessoais de idosos. Coimbra.

*Fratiglioni, L., Wang, H.-X., Ericsson, K., Maytan, M., \& Winblad, B. (2000). Influence of social network on occurrence of dementia: a community-based longitudinal study. The Lancet, 355(9212), 1315-1319. https://doi.org/10.1016/ S0140-6736(00)02113-9

Fung, H. H. \& Carstensen, L. L. (2006). Goals change when life's fragility is primed: Lessons learned from older adults, the September 11 attacks and SARS. Social Cognition, 24(3), 248-278. http://doi.org/10.1521/soco.2006.24.3.248

Gallagher, J., Luck, R. P., \& Del Vecchio, M. (2010). Case 1: Altered mental status - A state of confusion. Paediatrics and Child Health. https://doi.org/10.1521/soco.2006.24.3.248

Giles, L. C., Anstey, K. J., Walker, R. B., \& Luszcz, M. A. (2012). Social networks and memory over 15 years of followup in a cohort of older Australians: Results from the Australian longitudinal study of ageing. Journal of Aging Research, 2012. https://doi.org/10.1155/2012/856048

Jing, W., Willis, R., \& Feng, Z. (2016). Factors influencing quality of life of elderly people with dementia and care implications: A systematic review. Archives of Gerontology and Geriatrics, 66, 23-41. https://doi.org/10.1016/ j.archger.2016.04.009

*Jones, D. (2013). A family living with Alzheimer's disease: The communicative challenges. Dementia, 1-19. https:// doi.org/10.1177/1471301213502213

Kim, H. S., Sherman, D. K., \& Taylor, S. E. (2008). Culture and social support. American Psychologist, 63(6), 518-526. https://doi.org/10.1037/0003-066X

Kuiper, J. S., Zuidersma, M., Oude Voshaar, R. C., Zuidema, S. U., van den Heuvel, E. R., Stolk, R. P., \& Smidt, N. (2015). Social relationships and risk of dementia: A systematic review and meta-analysis of longitudinal cohort studies. Ageing Research Reviews, 22, 39-57. https://doi.org/10.1016/j.arr.2015.04.006

Simpson, C. \& Acton, G. (2013). Emotion work in family caregiving for persons with dementia. Issues in Mental Health Nursing, 34 (November), 52-8. https://doi.org/10.3109/01612840.2012.720649

*Smit, D. de Lange, J., Willemse, B., Twisk, J., \& Pot, A. M. (2016). Activity involvement and quality of life of people at different stages of dementia in long term care facilities. Aging \& Mental Health, 20(1), 100-109. https://doi.org/ 10.1080/13607863.2015.1049116

*Sørensen, L. V., Waldorff, F. B., \& Waldemar, G. (2008). Social participation in home-living patients with mild Alzheimer's disease. Archives of Gerontology and Geriatrics. https://doi.org/10.1016/j.archger.2007.08.011 
*Sörman, D. E., Rönnlund, M., Sundström, A., Adolfsson, R., \& Nilsson, L.-G. (2015). Social relationships and risk of dementia: a population-based study. International Psychogeriatrics C International Psychogeriatric Association, 278, 1391-1399. https://doi.org/10.1017/S1041610215000319

Tay, L., Tan, K., Diener, E., \& Gonzalez, E. (2013). Social Relations, Health Behaviors, and Health Outcomes: A Survey and Synthesis. Applied Psychology: Health and Well-Being, 5(1), 28-78. https://doi.org/10.1111/aphw.12000

*Wilson, R. S., Boyle, P. A., James, B. D., Leurgans, S. E., Buchman, A. S., \& Bennett, D. A. (2015). Negative Social Interactions and Risk of Mild Cognitive Impairment in Old Age HHS Public Access. Neuropsychology, 29(4), 561-570. http://doi.org/10.1037/neu0000154

*Wilson, R. S., Krueger, K. R., Arnold, S. E., Schneider, J. A., Kelly, J. F., Barnes, L. L., ..., \& Bennett, D. A. (2007). Loneliness and Risk of Alzheimer Disease, 64, 234-240. https://doi.org/10.1037/neu0000154

World Health Organization. (2012). Dementia: a public health priority. Dementia, 112. http://doi.org/978 9241564458

Wortmann, M. (2012). Dementia: a global health priority - highlights from an ADI and World Health Organization report. Alzheimer's Research \& Therapy, 4(5), 40. http://doi.org/10.1186/alzrt143

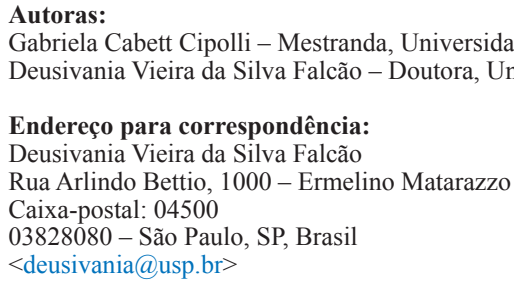

Recebido em: 21.12.2016

Aceito em: 24.04.2017 